\title{
A Study on Total Mixed Ration Feeding System for Feeding Pigs (1) - Development of Monorail Traveling TMR Feeder for Grow-Finish Pigs -
}

\author{
Hyuck Joo Kim ${ }^{1}$, Byeong Kee $\mathrm{Yu}^{1}$, Jong Tae Hong ${ }^{1}$, Kyu Hong Choi ${ }^{1}$, Ji Su Yu ${ }^{1}$, \\ Youngsin Hong', Yu Shin $\mathrm{Ha}^{2}$ \\ ${ }^{1}$ Farming Automation Division, National Academy of Agricultural Science, RDA., Suwon, Korea \\ ${ }^{2}$ Dept. of Bioindustrial Machinery Engineering, Kyungpook National University, Daegu, Korea
}

Received: November $4^{\text {th }}, 2013$; Revised: November $17^{\text {th }}, 2013$; Accepted: November $20^{\text {th }}, 2013$

\section{Abstract}

Purpose: Recent research showed that total mixed ration (TMR) feeding for pigs improved the productivity and reduced feed cost and manure odor. An automatic TMR feeding system was developed for this study because the conventional feeder cannot deliver the TMR containing roughage. Methods: Conventional feeding systems and physical properties of TMR were surveyed, and performance tests of the conventional feeder were conducted to develop a TMR feeder. Based on the TMR feeder was developed and installed, driving, measuring weight, radio frequency identification (RFID) reading, and discharging test for feeding were conducted to ensure the performance. Results: Moisture content, density, and angle of repose of the TMR 1 (mixture of $30 \%$ cut IRG silage and $70 \%$ concentrates) were $31.6 \%, 387 \mathrm{~kg} / \mathrm{m}^{3}$, and $51^{\circ}$, respectively. Moisture content, density, and angle of repose of the TMR 2 (mixture of $45 \%$ concentrates, 30\% cut IRG silage and byproducts, $10 \%$ bean curd refuse, $10 \%$ others, and $5 \%$ fermenter) were $22.2 \%, 544 \mathrm{~kg} / \mathrm{m}^{3}$, and $50^{\circ}$, respectively. The coefficient of variation (C.V.) of conventional concentrate feeding were 1.9 4.1\%, and C.V. of TMR containing 1 3\% cut IRG roughage feeding by conventional feeder were $9 \sim 42 \%$. The conventional disc type feeder was not suitable for TMR feeding because the supply unit was clogged. The C.V. of TMR 1 was $0.6 \sim 7.9 \%$ when $0.5 \sim 10 \mathrm{~kg}$ of the TMR supplied, and it was suitable for feeding grow-finish pigs and sows. On the contrary, the C.V. with TMR 2 was $28 \%$ when $0.5 \mathrm{~kg}$ of the TMR supplied, and it was not suitable for feeding sows. Conclusions: The TMR feeder developed in this study was suitable for feeding grow-finish pigs because the feeder performed stably with over $5.0 \mathrm{~kg}$ feed. However, the feeder showed a lack of accuracy for feeding sows because the amount of each feed was more than $0.5 \mathrm{~kg}$ per a feeding. Therefore, the improvement of outlet structure for accurate feeding is needed for sow feeding.

Keywords: By-products, Feeding pigs, Grow-finish pigs, Total mixed ration (TMR) feed system

\section{Introduction}

There is a high demand to improve productivity and reduce feed cost for livestock farms because the import cost for feed-corn has increased $\$ 305.89$ a ton in 2012 from \$157.47 in 2007 (Hyundai Research Institute, 2012) and external environment has deteriorated due to the Korea-US FTA and Korea-EU FTA. Since feed cost takes

\footnotetext{
*Comesponding author: Hyuck Joo Kim

Tel: +82-31-290-1867; Fax: +82-31-290-1860

E-mail: agrihj@rda.go.kr
}

the major part in farm administration, the cattle and dairy farmers have fed total mixed ration (TMR) using various foodstuffs as well as concentrates.

Japan's current agricultural policy plan (Food, Agriculture and Rural Areas Basic Plan) and food recycling law propel the eco feed plan, which use the agricultural by-products, food by-products, and restaurant leftover to make concentrates in terms of recycling resources (MAFF, 2012). Researchers in Japan have made progress toward TMR feed, and Shimazawa (2009) studied on high-quality pork production using local agricultural by- 
product. European countries have used by-products to make concentrates, especially, feeding liquid by-products or feeding high-moisture maize grain by-products were used for feeding grow-finish pigs (Gatel et al., 1988). Monorail type feeder was used to deliver roughage to the animals (WATTAgNet. 2008).

The research (Cho, 2009) from National Academy of Agricultural Science introduced that the improved productivity of pig production, reduced stress, reduced manure odor, improved immunity were reported after feeding a fermented TMR. The fermented TMR for feeding sows increased PSY (Produced piglets per a Sow per a Year) by one, and a combination of $10 \%$ Italian ryegrass (IRG) pellet was suitable for feeding sows (Kim et al, 2012a). The TMR feed system in pig farms using agricultural by-products (e.g. roughage) and food by-products should be developed in order to reduce feed cost and increase productivity of pig production. In Korea, there were 100 TMR centers where TMR was made and sold, but all of them were for cattle or cows (NIAS, 2009). Though TMR feeding systems for cattle have already disseminated widely, they depended heavily on the imported ones. In Korea, advanced automatic feeding systems for each animal have not developed yet; as a result, the TMR feeding systems are heavily dependent on the imported ones. Since it is difficult to adopt the TMR feeder for cattle or cows into pig feeding, farmers working at pig farms mixed the concentrates with by-products and roughage and distributed to the pigs, which is no longer possible due to the lack of labor (Kim et al., 2012b).

The purpose of this study was to develop a TMR feed system for feeding pigs to increase productivity and reduce feed cost in the pig farms. This study surveyed the factors influencing on the feeder to develop a feeding system and conducted performance tests for the prototype TMR feeder.

\section{Materials and Methods}

\section{Pig feeding system in Korea}

This study surveyed the pig feeding systems to explore the possibility of the conventional feeder for pellet and TMR feeding and to establish a model of TMR feeding system. The survey was conducted at three sample farms in 2011. As shown in Figure 1, dry feeders for concentrates and pellet feeding were used for pregnant sows, farrowing sows, and grow-finish pigs. Mixture of concentrates and pellet can be used with the conventional dry feeder (disc chain or disc wire type). The mixture was delivered by the disc wire or chain inside the pipe at the conventional feeder as shown in Figure 1(a). This type is suitable for the concentrates with low moisture content and small particle, and not suitable for the concentrates with high moisture content $(20 \%$, w.b.) and lots of roughage.

Most countries used the conventional dry feeder, the same with the one in Korea for dry concentrates, and monorail type feeder operated by a computer also used as in Figure 2 (PELLON, 2007). However, this monorail feeder is suitable for dry concentrates not for TMR. There is no TMR feeder in the pig farms except the one in Figure 3 which delivers roughage using monorail type feeder (WATTAgNet. 2008).

Therefore, this study conducted tests of TMR delivery and feeding using the conventional feeder. From the results of the test, monorail type was appropriate for the TMR feeder, and the prototype was designed for this study.

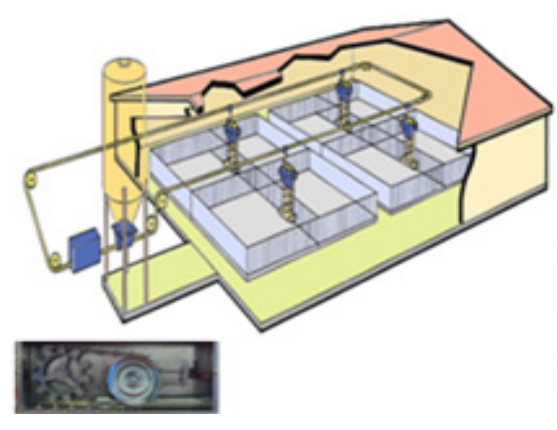

(a) Dry feeder system

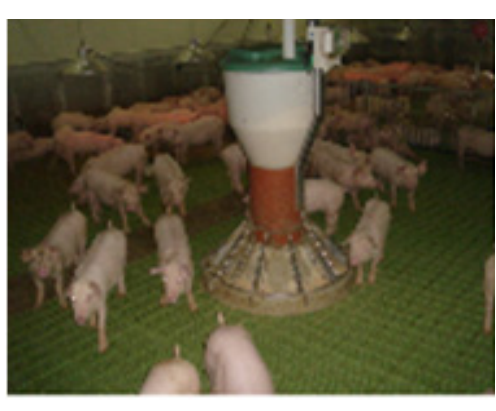

(b) Grow-finish pigs

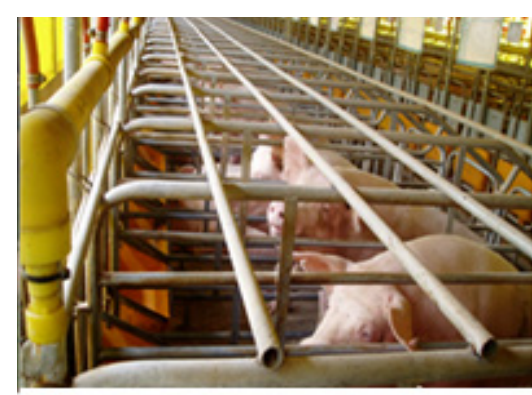

(c) Pregnant sow

Figure 1. Dry concentrate feeder. 


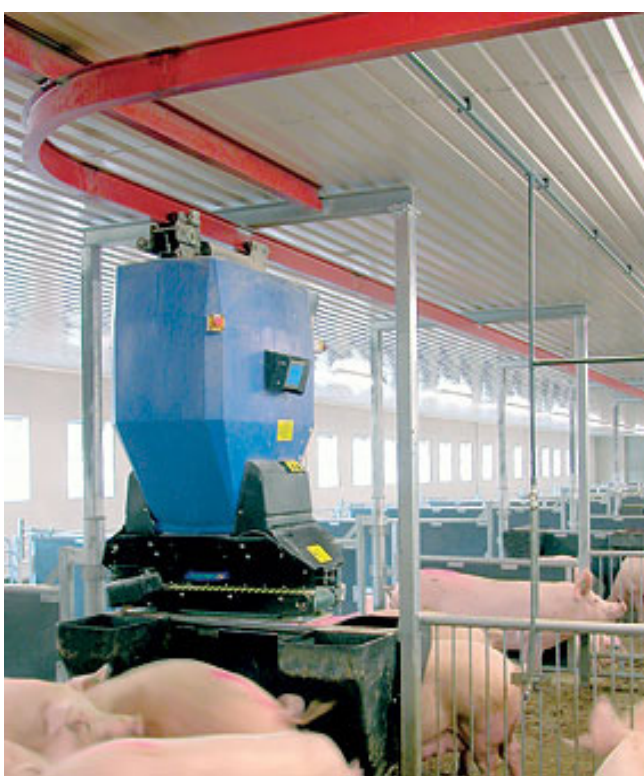

Figure 2. Monorail type.

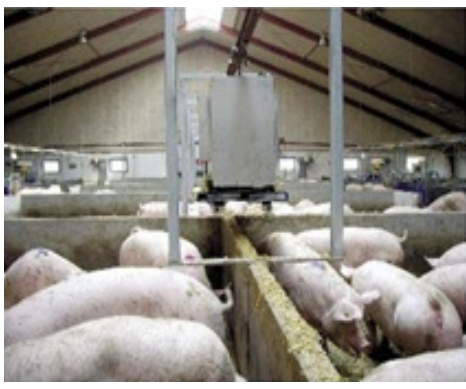

(a) Straw supply

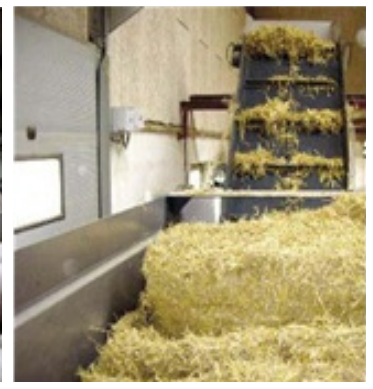

(b) Monorail type feeder dry concentrate feeder
Figure 3. Straw feeder.

\section{Physical properties of TMR}

TMR is a mixture of various feedstuffs, so the physical properties of it differ depending on the input materials. The sample was the mixture of concentrates and cut IRG silage, and "omega 3 TMR for pigs" was made at G pig farm located in Chungnam Province. Moisture content, density, and angle of repose of the TMR were analyzed, and the results were utilized in designing a TMR feeding system.

\section{TMR 1}

The concentrates and fermented IRG silage were mixed with certain mixing ratio for feeding TMR. According to Kim et al (2012), adding 10\% IRG silage pellet (moisture content 15\%, w.b.) increased the productivity of sows, and adding $20 \%$ and $30 \%$ also had the same effect. Additionally, there is a report (Cho, 2012) that

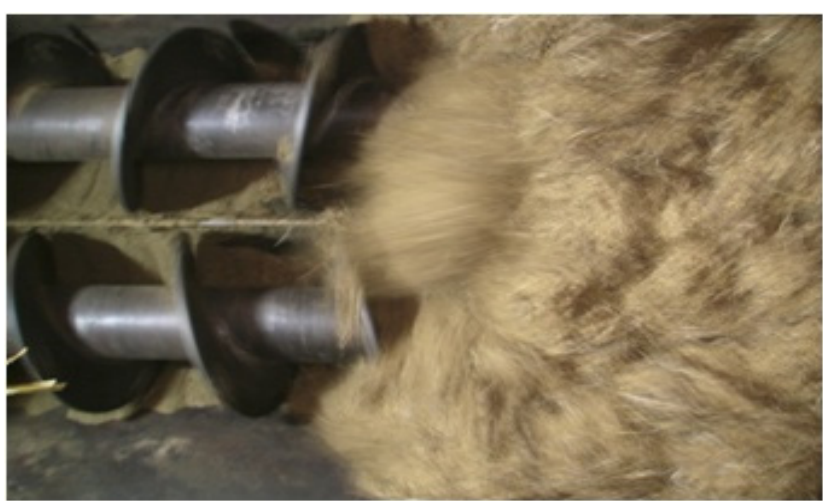

Figure 4. TMR Mixing (concentrates $70 \%$ + cut IRG silage 30\%).

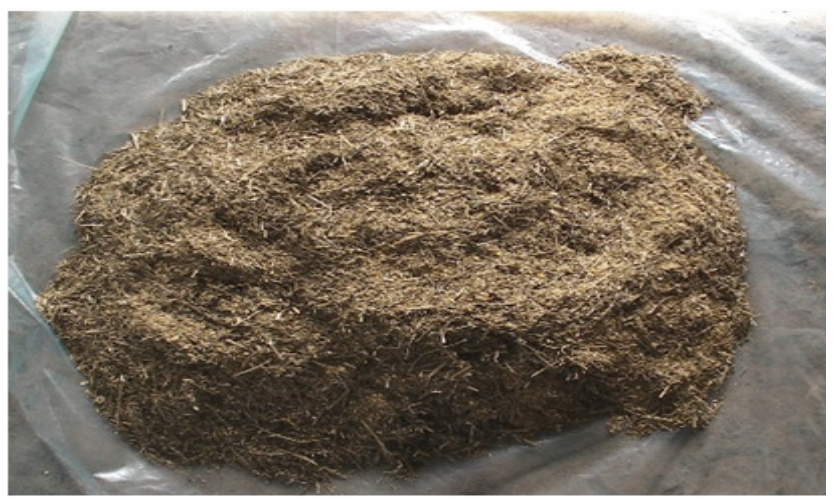

Figure 5. Mixed TMR 1 (concentrates 70\% + cut IRG silage 30\%).

TMR feeding including 1 1.5\% of dried IRG powder could decrease the phenols and indols in pig feces which cause odors from pig farms. Therefore, in this study, we used 30\% IRG silage (moisture content 60\%, w.b.) for feeding sows and 5\% IRG silage for feeding grow-finish pigs. Figure 4 shows the mixing of IRG silage cut by $3-\mathrm{cm}$ and concentrates. After unrolling the round bale silage, the silage was cut and input into the mixer in order to mix with concentrates. Figure 5 shows mixed TMR 1 of $30 \%$ cut IRG silage and $70 \%$ concentrates.

\section{TMR 2}

The experimental site, G pig farm has fed pigs using by-products from food and pharmaceutical company for antibiotic-free pig breeding. The second sample, TMR 2 was made with $45 \%$ concentrates, $30 \%$ cut IRG silage and by-products, $10 \%$ bean curd refuse, $10 \%$ others, and $5 \%$ fermenter as shown in Figure 6.

\section{Moisture content, density, angle of repose}

Measurement of moisture content with $5 \mathrm{~g}$ of each sample, TMR 1 and 2 using a moisture analyzer (MX-50, 


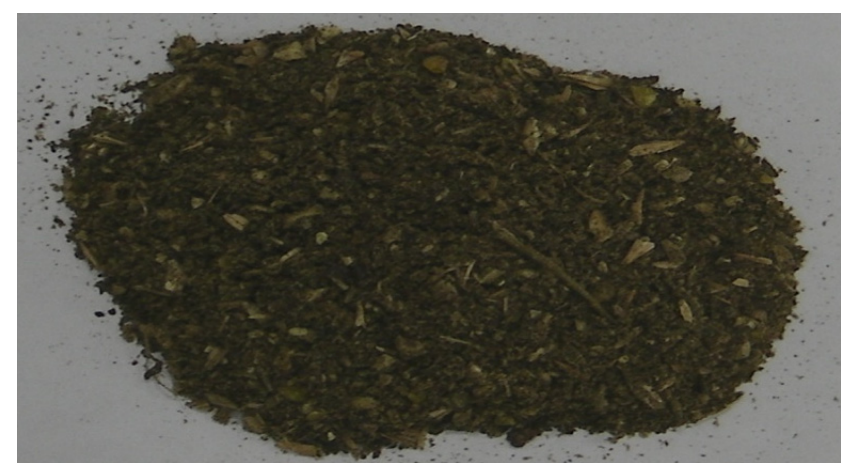

Figure 6. TMR 2 (concentrates $45 \%+$ cut IRG silage and by-products $30 \%+$ bean curd refuse $10 \%+$ others $10 \%+$ fermenter $5 \%$ ).

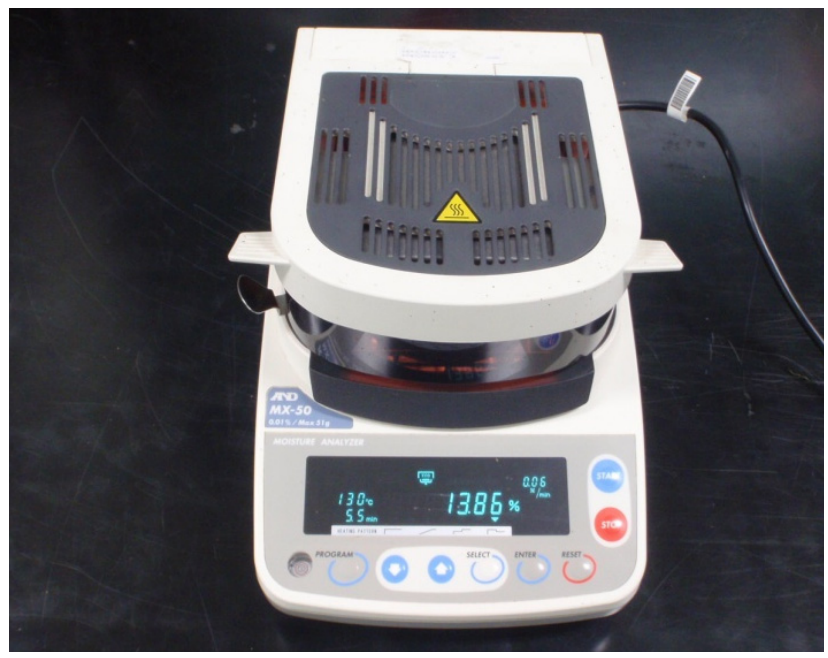

Figure 7. Measurement of moisture contents.

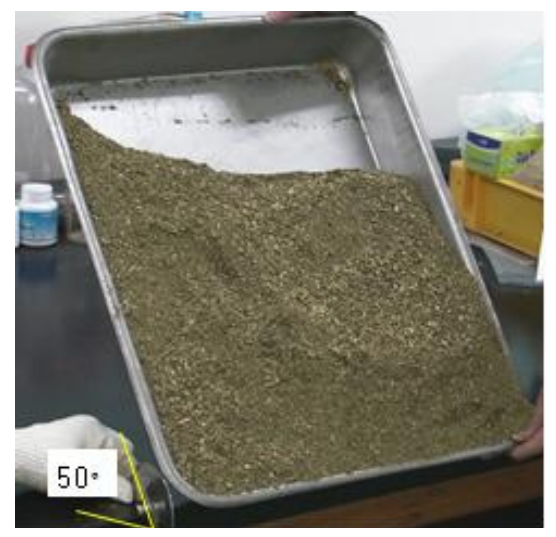

Figure 8. Measurement of repose angle.

Tokyo, Japan) as shown in Figure 7 repeated three times. For measurement of density, $1000 \mathrm{~mL}$ of each sample was placed in a beaker and measured three times, and the unit for density was $\mathrm{kg} / \mathrm{m}^{3}$. Each sample was filled in a rectangular tray $(540 \times 420 \times 50 \mathrm{~mm})$ flat up to the height of the tray, and then the angle of repose was measured at the angle just before the sample flow out when the tray was raised. The angle of repose measurement was repeated three times (Figure 8).

\section{Feeding experiment using a conventional disc chain feeder for concentrate feed and TMR}

Experiment with the conventional disc chain was conducted to know the accuracy of conventional feeding and to see the availability of the TMR feeding. The disc chain feeder (Figure 9) consists of drive unit (motor driven), hopper (input concentrates), feeding pipe, corner (delivering concentrates, diameter of $60 \mathrm{~mm}$ ), and drop feeder (volumetric distribution). In this experiment, the TMR inputted at the hopper (Figure 10) was delivered to the drop feeder via feeding pipe, and the drop feeder dropped down the feed onto the feeding table. Drop feeder in the fifth place of Figure 9 had a stop sensor for automatic controlling. The coefficient of variation (C.V.) and average of the concentrate and TMR dropped from drop feeders were measured. The roughage rate of the material (combination of concentrates and IRG

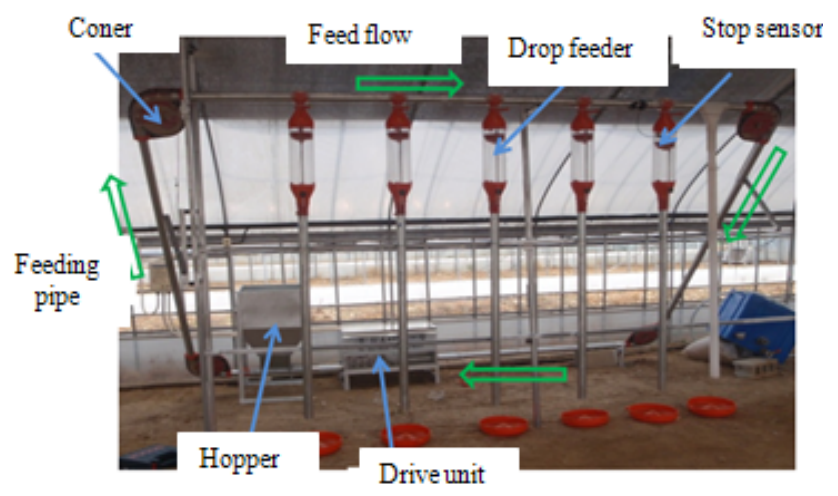

Figure 9. Conventional feeding system.

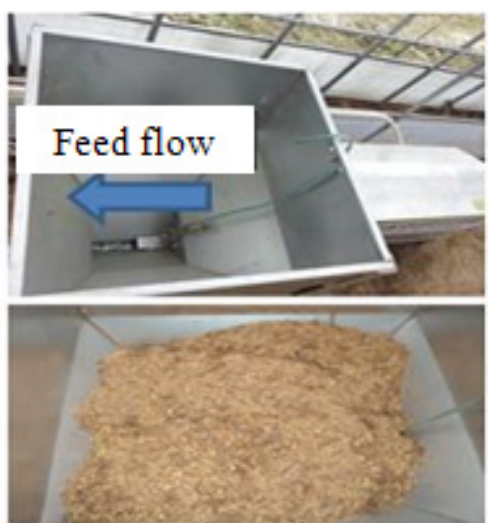

Figure 10. Input TMR. 
silage) was $1 \%, 2 \%$, and $3 \%$ in dry matters, and the length of the cut IRG silage was average $37.3 \mathrm{~mm}$ (standard deviation (S.D.), $16 \mathrm{~mm}$ ).

\section{Experiment of TMR feeding using a $1^{\text {st }}$ prototype of TMR feeder}

Figures 11 and 12 show diagram and photo of the monorail type TMR feeder fabricated for experimental testing. The dimensions of the device were $1300 \times 2487$ $\times 507 \mathrm{~mm}$, and the inner space was designed to have volume of $1 \mathrm{~m}^{3}$ and maximum load of $150 \mathrm{~kg}$ because the average density of the TMR 1 was $387 \mathrm{~kg} / \mathrm{m}^{3}$. Motor-

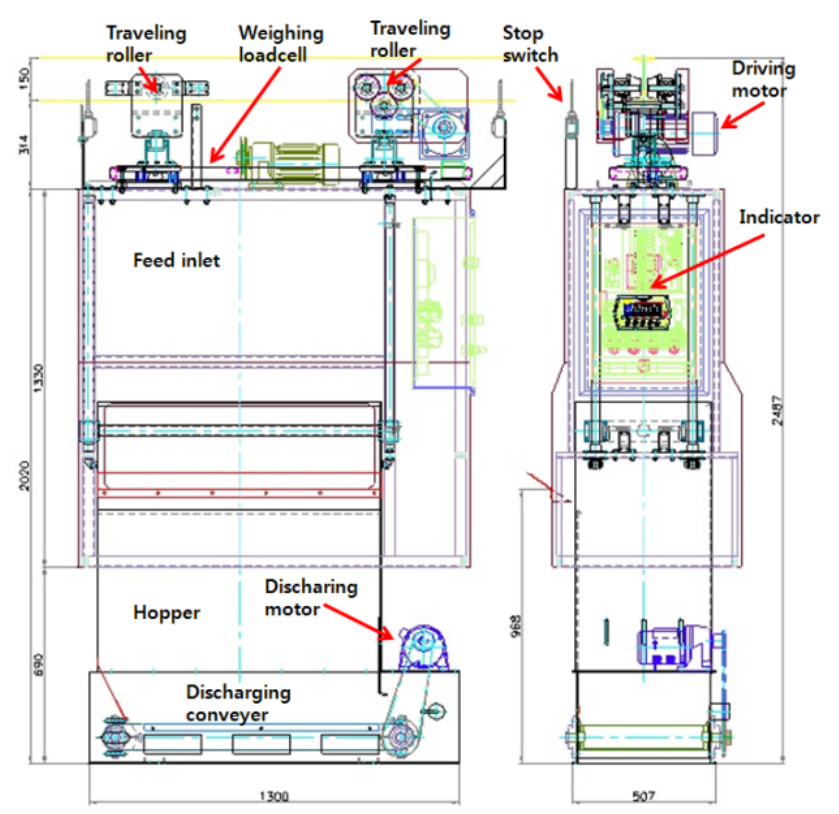

Figure 11. Diagram of $1^{\text {st }}$ prototype.

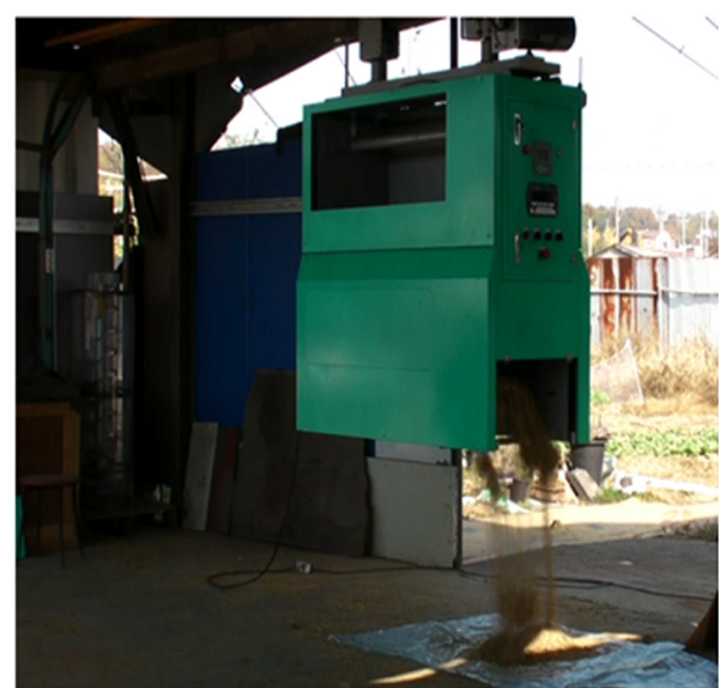

Figure 12. Photo of $1^{\text {st }}$ prototype. driven wheels were driving on the monorail, and the load was hung on the loadcell located on the upper part of the rail. The driving and discharging were controlled automatically by time sequence control system with one-board micom and loadcell weight sensing. The power source used a single-phase $220 \mathrm{~V} \mathrm{AC}$ motor, and the power line moved along the feeder. The internal shape of the hopper was vertical in order to drop the TMR feed containing lots of roughages down regardless the angle of repose. The TMR feed was discharged on either side of the belt by the motor-driven belt conveyor. The discharging rate can be controlled by adjusting feeding times or feed amount from loadcell.

The sample was the mixture of IRG silage cut by $3-\mathrm{cm}$ of TMR 1 (cut IRG silage 30\% + concentrates 70\%). The discharging rate in a time sequence control system and loadcell weight sensing and C.V. of discharging rate were measured. After TMR was inputted at the hopper, the discharging rate by time and weight were set in the input device. And then, the TMR feed was discharged by the motor-driven belt conveyor. The discharging rate in a time sequence control system was measured by setting drive distance and discharging time until 15 sec with interval of $5 \mathrm{sec}$ after inputting TMR at the hopper. The discharging rate in a loadcell weight sensing system was measured by setting discharge weight until $15 \mathrm{~kg}$ with intervals of $5 \mathrm{~kg}$.

\section{Experiment using a final prototype of monorail traveling TMR feeder}

Results of experimental testing of $1^{\text {st }}$ prototype showed that discharging of large amount of feed had no problem, but small amount of feed had accuracy problems. In addition, the cost for installing rail was expensive because the device needed large-scale rail owing to its heavy weight. As a result, final prototype of monorail traveling TMR feeder was designed to decrease its size and to increase the accuracy as in Figure 13. Figure 14 shows the controller block diagram of the feeder.

The final prototype TMR feeder was reduced the size more than half of the $1^{\text {st }}$ prototype to the dimensions of $700 \times 600 \times 1,428 \mathrm{~mm}$. The maximum load was 70 $\mathrm{kg}$, and hanger type two-wheel driven monorail made with round pipe of $100 \mathrm{~mm}$ diameter was used. The reader attached on the feeder recognized the drive position automatically by reading the RFID card inserted on the rail. The limit sensor was installed in the feeder 
on the ground in order to feed TMR automatically, and controller received signals if the feeder was empty. When the controller recognized the weight and RFID position from the load cell, TMR feeder start and deliver feed based on the speed and feed rate inputted by an operator. As in Figure 14, the automatic controller of the feeder consisted of MPU (Microprocessor unit) with the operating firmware program, RFID module for recognizing position, motor driver for traveling and feeding, wireless zigbee module for sending and receiving signals with CPU, command input device, and LCD display. Built-in DC $24 \mathrm{~V}$ battery was used for the power; the charging device was installed at the starting point of feeder, and the feeder always charged at the starting point in case it was not working.

The TMR feed was discharged by the motor-driven belt conveyor. As shown in Figure 13, rotating roller was installed inside the hopper, and the conveyor was installed at the bottom. However, frequent clogging occurred

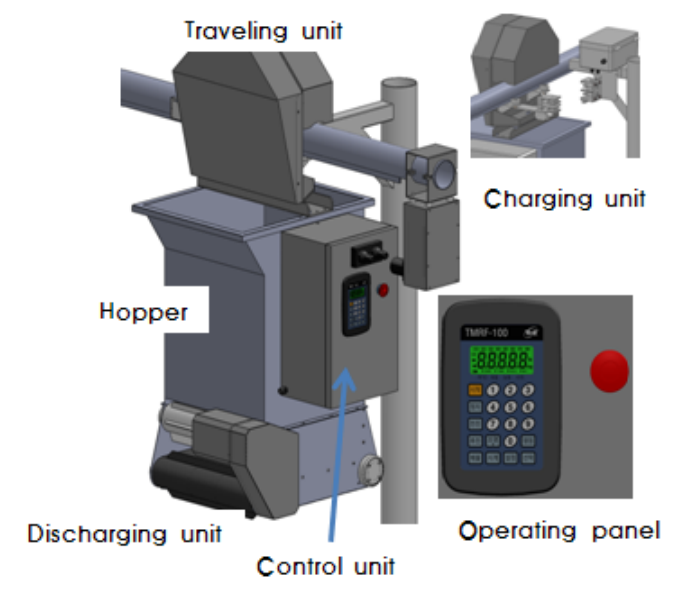

Figure 13. Drawings of final prototype.

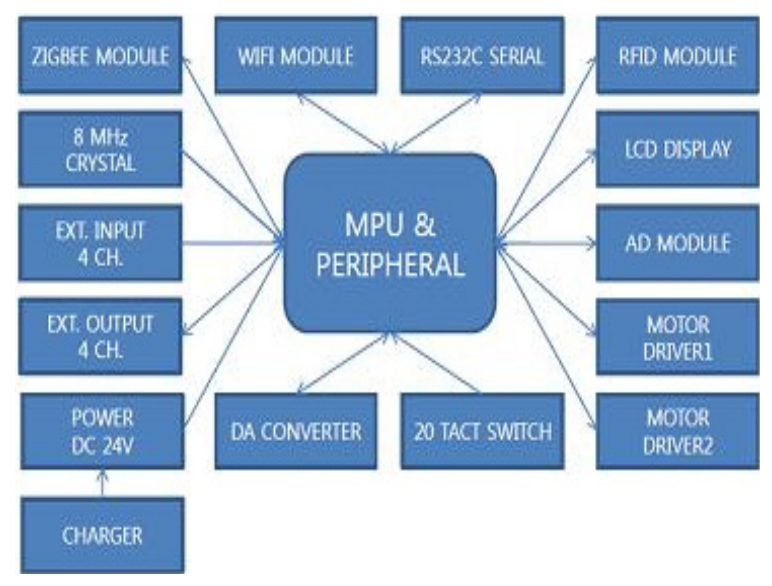

Figure 14. Block diagram of controller. with high roughage content. Therefore, extra conveyor was installed in opposite directions of discharging conveyor inside the hopper in order to solve the clogging. Slot at the top of the hopper was slanted to $60^{\circ}$ to input TMR feed avoiding the interference of pipe rail. The input device set the speed of TMR feeder, discharge rate, current weight, and number of RFID.

Figure 15 shows the experiment with final prototype feeder. The experiment was started with loading the feed at the hopper. After loading the TMR in the feeder, it travels the monorail till the RFID reader read the RFID card attached on the pipe. And then the feeder discharge the specified amount of TMR feed to the ground feeder and returned to the starting point The limit switch stopped the returned feeder at the starting point, and the charging began by the contact on the charging device. The distance

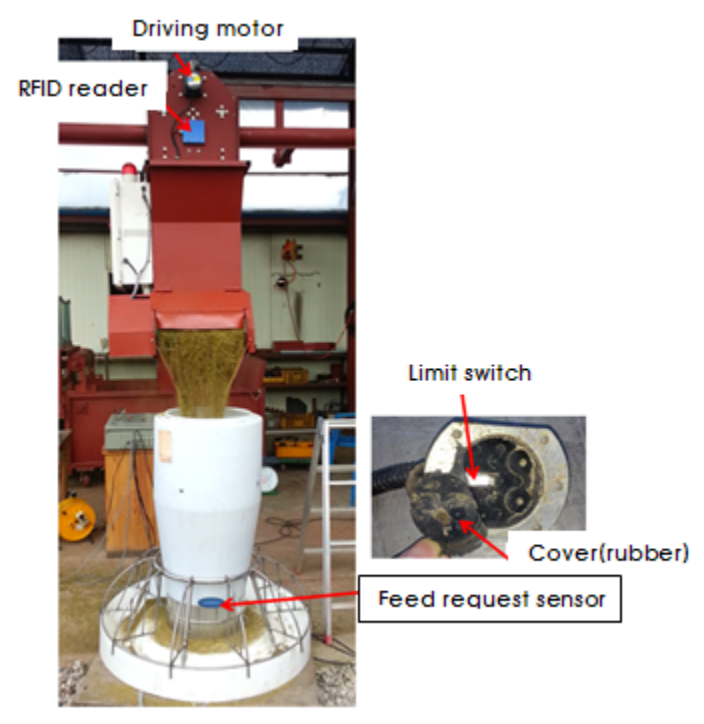

Figure 15. Feeding experiment.

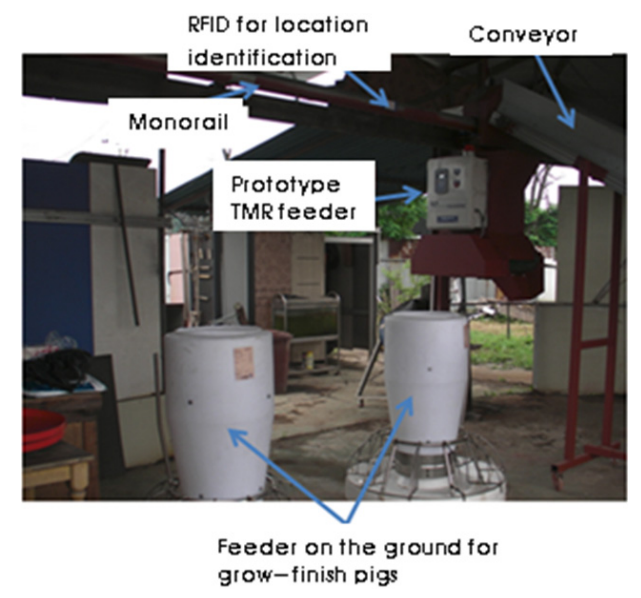

Figure 16. Performance test of prototype TMR feeder. 
between the charging unit and the ground feeder were $3.2 \mathrm{~m}$, and the measurement of speed repeated three times. The weight added in the prototype was measured within the error range using four different weights $(12.30$ $\mathrm{kg}, 19.98 \mathrm{~kg}, 36.29 \mathrm{~kg}, 55.55 \mathrm{~kg}$ ). The S.D. (standard deviation) and C.V. (coefficient of variation) per feed rate of each material, TMR 1 and TMR 2 were measured to test the performance of the feeder.

\section{Results and Discussions}

\section{Physical properties of the TMR}

Table 1 shows the physical properties of TMR 1 . Average moisture content of it was $31.6 \%$ (w.b.), which was lower than the one of haylage (40 60\%, w.b.). Average density was $387 \mathrm{~kg} / \mathrm{m}^{3}$, and it became lower when the roughage rate increased. The measured physical properties were used to design the feeder.

Table 2 shows the physical properties of TMR 2 .
Average moisture content was $22.2 \%$ (w.b.), which was lower than the one of TMR 1 . Average density was 544 $\mathrm{kg} / \mathrm{m}^{3}$, which was higher than the one of TMR 1 due to the less amount of IRG silage.

\section{Experiment of TMR feeding using conventional disc chain type}

Table 3 shows the results of the experiment of conventional concentrate feeding by conventional disc chain type feeder. The C.V. of feeding amount was $1.9 \sim 4.1 \%$. Therefore, it would be appropriate that the C.V. of feeding amount of prototype feeder could be less than $5.0 \%$.

Table 4 shows the results of the experiment with conventional disc chain. The C.V. of the roughage was 9 42\%, and the inlet was clogged because of the roughage (Figure 17). Modification of the hopper needed to solve the clogging. For that reason, the hopper was enlarged, and an auger feeder was added in it (Figure 18).

Table 5 shows the result of the experiment with the

Table 1. Physical properties of TMR 1 (concentrates 70\% + cut IRG silage 30\%)

\begin{tabular}{cccc}
\hline Replication & Moisture content $(\%, w . b)$. & Density $\left(\mathrm{kg} / \mathrm{m}^{3}\right)$ & Angle of repose $\left({ }^{\circ}\right)$ \\
\hline 1 & 29.5 & 400 & 53 \\
2 & 31.2 & 380 & 49 \\
3 & 34.2 & 380 & 51 \\
Average & 31.6 & 387 & 51 \\
\hline
\end{tabular}

Table 2. Physical properties of TMR 2 (concentrates $45 \%+$ cut IRG silage and by-products $30 \%+$ bean curd refuse $10 \%+$ others $10 \%+$ fermenter $5 \%)$

\begin{tabular}{cccc} 
Replication & Moisture content $(\%$, w.b.) & Density $\left(\mathrm{kg} / \mathrm{m}^{3}\right)$ & Angle of repose $\left(^{\circ}\right)$ \\
\hline 1 & 22.29 & 543 & 47 \\
2 & 22.23 & 526 & 52 \\
3 & 22.08 & 564 & 50 \\
Average & 22.2 & 544 & 50 \\
\hline
\end{tabular}

Table 3. C.V. and average of feeding conventional concentrate feed

\begin{tabular}{|c|c|c|c|c|c|}
\hline \multirow{2}{*}{ Replication } & \multirow{2}{*}{ Feeding time } & \multicolumn{4}{|c|}{ Feeding amount } \\
\hline & & $\# 1$ & \# 2 & $\# 3$ & \# 4 \\
\hline 1 & 68.0 & 1325.0 & 1225.0 & 1337.0 & 1267.0 \\
\hline 2 & 65.0 & 1387.0 & 1287.0 & 1392.0 & 1395.0 \\
\hline 3 & 59.0 & 1401.0 & 1267.0 & 1395.0 & 1384.0 \\
\hline 4 & 67.0 & 1384.0 & 1289.0 & 1352.0 & 1367.0 \\
\hline 5 & 63.0 & 1395.0 & 1201.0 & 1386.0 & 1402.0 \\
\hline Average & 64.4 & 1378.4 & 1253.8 & 1372.4 & 1363.0 \\
\hline \multicolumn{2}{|c|}{ C.V. (\%) } & 2.2 & 3.1 & 1.9 & 4.1 \\
\hline
\end{tabular}


Kim et al. A Study on Total Mixed Ration Feeding System for Feeding Pigs (1) - Development of Monorail Traveling ... Journal of Biosystems Engineering • Vol. 38, No. 4, 2013・www.jbeng.org

Table 4. C.V. and average roughage rate in a TMR feed

\begin{tabular}{|c|c|c|c|c|c|c|}
\hline \multirow{2}{*}{ Drop feeder } & \multicolumn{2}{|c|}{ Roughage rate $1 \%$} & \multicolumn{2}{|c|}{ Roughage rate $2 \%$} & \multicolumn{2}{|c|}{ Roughage rate $3 \%$} \\
\hline & Average (\%) & C.V. (\%) & Average (\%) & C.V. (\%) & Average (\%) & C.V. (\%) \\
\hline$\# 1$ & 0.51 & 12 & 1.02 & 38 & 1.29 & 10 \\
\hline$\# 3$ & 1.00 & 12 & 1.78 & 26 & 2.70 & 17 \\
\hline$\# 4$ & 0.95 & 17 & 1.98 & 9 & 2.69 & 42 \\
\hline
\end{tabular}

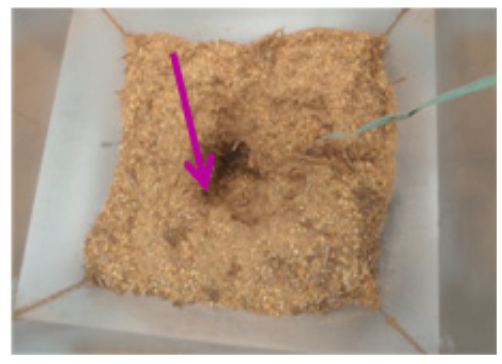

Figure 17. Clogging at inlet.

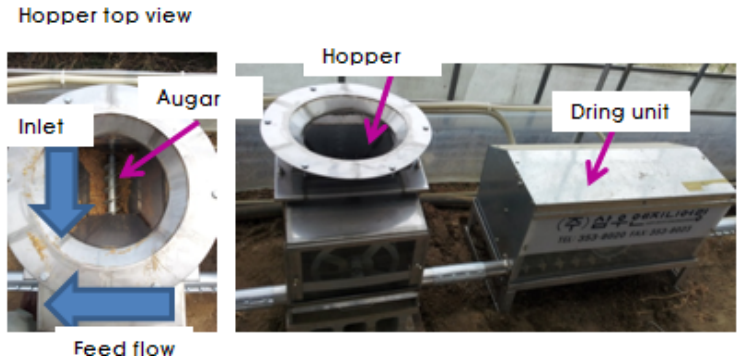

Figure 18. Revised hopper.

Table 5. C.V. and average roughage rate with revised hopper

\begin{tabular}{cccccccc} 
& \multicolumn{2}{c}{ Roughage rate $1 \%$} & \multicolumn{2}{c}{ Roughage rate $2 \%$} & \multicolumn{3}{c}{ Roughage rate $3 \%$} \\
\cline { 2 - 7 } Drop feeder & Average (\%) & C.V. (\%) & Average (\%) & C.V. (\%) & Average (\%) & C.V. (\%) \\
\# 3 & 1.34 & 34 & 1.66 & 10 & 2.59 & 7 \\
\# 4 & 0.95 & 5 & 1.82 & 19 & 2.65 & 14 \\
\hline
\end{tabular}

Table 6. Discharging rate in a time sequence control system

\begin{tabular}{ccccccc} 
Time $(\mathrm{sec})$ & \multicolumn{5}{c}{ Discharging weight $(\mathrm{kg})$} \\
\cline { 2 - 7 } & $\# 1$ & $\# 2$ & $\# 3$ & Average & S.D. & C.V. \\
10 & 3.0 & 4.0 & 7.2 & 4.7 & 2.2 & 46.8 \\
15 & 9.0 & 6.2 & 6.9 & 7.4 & 1.5 & 20.2 \\
\hline
\end{tabular}

Table 7. Discharging rate in a weight feedback control system

\begin{tabular}{cccccccc} 
Control weight $(\mathrm{kg})$ & \multicolumn{9}{c}{ Discharging weight $(\mathrm{kg})$} \\
\cline { 2 - 7 } & $\# 1$ & $\# 2$ & $\# 3$ & Average & S.D. & C.V. \\
10 & 5.5 & 4.8 & 5.2 & 5.2 & 0.4 & 7.7 \\
15 & 10.7 & 11.0 & 10.2 & 10.6 & 0.4 & 3.8 \\
\hline
\end{tabular}

revised hopper. The C.V. of the roughage rate from $1 \sim 3 \%$ was $4 \sim 34 \%$, which was difficult to distribute feeding evenly. In addition, the feeder worked well with the roughage rate $1 \sim 2 \%$, but roughage rate $3 \%$ showed clogging or congestion in the transfer line. Therefore, the conventional disc chain feeder was not suitable for the TMR feed due to the clogging and wide range of C.V.
$(4 \sim 34 \%)$.

\section{Experiment of TMR feeding using a $1^{\text {st }}$ prototype of TMR feeder}

Discharging rate in a time sequence control system was measured at an interval of 5 seconds between 5 and 15 seconds (Table 6). The rate was not uniform, 
so the discharging rate control system using a weight feedback was needed. Discharging rate in weight feedback control was measured at 5, 10, and $15 \mathrm{~kg}$, and the C.V. was $3.8 \sim 7.7 \%$ (Table 7). It had no problems in feeding grow-finish pigs, but the feed under $5 \mathrm{~kg}$ was not suitable. Some modifications to improve accuracy still needed.

\section{Experiment using a final prototype of monorail traveling TMR feeder}

Final prototype of TMR feeder had two RFID cards: one on the base, the other on the feeder. The drive distance was $3.2 \mathrm{~m}$ from the charging point to feeder, and the average travel speed was $0.33 \mathrm{~m} / \mathrm{s}$ (Table 8).

Error range of the measured weight was $\pm 0.1 \mathrm{~kg}$, and the proper loading was $55 \mathrm{~kg}$ in the feeder having maximum load of $70 \mathrm{~kg}$ (Table 9).

Standard deviation of setting discharging rate per unit from $0.5 \mathrm{~kg}$ to $10 \mathrm{~kg}$ with TMR 1 was $0.03 \sim 0.27$, and C.V. was $0.6 \sim 7.9 \%$ (Table 10). The results showed that the feeder was suitable for feeding. When the discharging

Table 8. Monorail traveling speed

\begin{tabular}{|c|c|c|}
\hline Drive distance $(\mathrm{m})$ & Time (s) & Speed $(\mathrm{m} / \mathrm{s})$ \\
\hline \multirow{5}{*}{3.2} & 9.8 & 0.33 \\
\hline & 10.0 & 0.32 \\
\hline & 9.7 & 0.33 \\
\hline & 9.6 & 0.33 \\
\hline & 9.6 & 0.33 \\
\hline Average & 9.7 & 0.33 \\
\hline
\end{tabular}

Table 9. Accuracy of weight sensing

\begin{tabular}{ccccc} 
Weight $(\mathrm{kg})$ & Measured weight $(\mathrm{kg})$ & \multicolumn{2}{c}{ Error } \\
\cline { 3 - 5 } & & $(\mathrm{kg})$ & $(\%)$ \\
12.30 & 12.2 & 0.10 & -0.8 & -0.9 \\
19.98 & 19.8 & 0.18 & -0.8 & -0.3 \\
\hline 36.29 & 36.0 & 0.29 & 0.15 & -0.3 \\
\hline
\end{tabular}

Table 10. Discharging rate of TMR 1 by final prototype feeder

\begin{tabular}{ccccc} 
Control weight $(\mathrm{kg})$ & Discharging weight $(\mathrm{kg})$ & Average $(\mathrm{kg})$ & S.D. & C.V. $(\%)$ \\
\hline 0.5 & $0.78 / 0.76 / 0.72$ & 0.75 & 0.03 & 4.0 \\
1 & $1.08 / 1.22 / 1.1$ & 1.13 & 0.08 & 6.6 \\
3 & $2.94 / 3.06 / 3.42$ & 3.14 & 0.25 & 7.9 \\
5 & $5.06 / 5.10 / 5.04$ & 5.06 & 0.03 & 0.6 \\
7.5 & $7.30 / 7.64 / 7.84$ & 7.59 & 0.27 & 3.6 \\
10 & $9.98 / 9.98 / 9.78$ & 9.88 & 0.10 & 1.0 \\
\hline
\end{tabular}

Table 11. Discharging rate of TMR 2 by final prototype feeder

\begin{tabular}{ccccc} 
Control weight $(\mathrm{kg})$ & Discharging weight $(\mathrm{kg})$ & Average $(\mathrm{kg})$ & S.D. & C.V. $(\%)$ \\
\hline 0.5 & $0.56 / 0.96 / 0.96$ & 0.82 & 0.23 & 28.0 \\
1 & $1.26 / 1.36 / 1.36$ & 1.32 & 0.05 & 4.0 \\
3 & $3.76 / 3.56 / 3.76$ & 3.69 & 0.11 & 3.0 \\
5 & $5.76 / 5.96 / 5.76$ & 5.82 & 0.11 & 1.0 \\
7.5 & $8.76 / 8.56 / 8.56$ & 8.62 & 0.11 & 1.0 \\
10 & $11.16 / 11.16 / 11.00$ & 11.10 & 0.09 & 0.8 \\
\hline
\end{tabular}


rate was set $1 \sim 10 \mathrm{~kg}$ with TMR 2, C.V. was $0.8 \sim 4 \%$ which proves no problem in feeding (Table 11). However, when the discharging rate was set $0.5 \mathrm{~kg}$, C.V. was $28 \%$ which needs modification of the feeder because the discharging time was set for a short period with little discharging rate, then the extra feeding could occurred due to the inertia.

The feeder developed for this study performed stably in feeding over $5.0 \mathrm{~kg}$ which shows the C.V. below 5 $\%$; therefore, it was suitable for feeding grow-finish pigs which need more than $10 \mathrm{~kg}$ of feeds per a feeding. However, the feeder was not suitable for feeding sows which needs minimum $0.5 \mathrm{~kg}$ per a feeding.

\section{Conclusions}

There have been needs for developing a TMR feed system for growing pigs to increase productivity and reduce odor from feces in the pig farms. Therefore, conventional feeding systems and physical properties of the TMR were researched to develop a TMR feed system. Based on a TMR feeder was developed and installed to test the performance. The results of this study were as follows:

(1) The disc chain or disc wire type dry feeder was used for feeding pregnant sows, farrowing sows, and grow-finish pigs in most pig farms. The pellet was suitable for the conventional feeder, but the TMR feeds would not be suitable for the conventional feeder.

(2) TMR 1 was the mixture of $30 \%$ cut IRG silage and $70 \%$ concentrates. The moisture content, density, and angle of repose of it were $31.6 \%, 387 \mathrm{~kg} / \mathrm{m}^{3}$, and $51^{\circ}$, respectively. TMR 2 was made with $45 \%$ concentrates, 30\% cut IRG silage and by-products, $10 \%$ bean curd refuse, $10 \%$ others, and $5 \%$ fermenter. The average moisture content, density, and angle of repose of TMR 2 were $22.2 \%, 544$ $\mathrm{kg} / \mathrm{m}^{3}$, and $50^{\circ}$, respectively.

(3) At experiment of conventional concentrate feeding by conventional disc chain type feeder, the C.V. of feeding amount was $1.9 \sim 4.1 \%$. On the other hand, in TMR feeding by the conventional feeder, the C.V. of roughage was $9 \sim 42 \%$, and the supply part was clogged. Even though modifications such as enlarging the supply part and adding auger feeder were made to solve the problems, the supply part was clogged at a roughage rate of $3 \%$. In addition, the C.V. of TMR was $4 \sim 34 \%$. Considering all of these, the conventional feeder was not suitable for TMR feeding.

(4) Measurement of discharging rate in a time sequence control system of $1^{\text {st }}$ prototype showed that C.V. was $20.2 \sim 47.5 \%$. Therefore, time control was not suitable for feeding due to the uneven discharging rate. Meanwhile, C.V. was $3.8 \sim 7.7 \%$ at the measurement of discharging rate in a weight feedback control system, which means that the weight control feeder had no problem for feeding growfinish pigs. The test results showed that discharge of large amount of feed had no problem, but small amount of feed below $5.0 \mathrm{~kg}$ had accuracy problems. Furthermore, the cost for installing rail was expensive because it needed large-scale rail due to its heavy weight.

(5) On the basis of previous survey and test, the final prototype monorail traveling TMR feeder was developed and tested. The results showed that the C.V. of TMR 1 (mixture of $30 \%$ cut IRG silage and $70 \%$ concentrates) was $0.6 \sim 7.9 \%$ when $0.5 \sim 10 \mathrm{~kg}$ of the TMR supplied, which was suitable for feeding pigs. In contrast, the C.V. of feeding TMR 2 was $28 \%$ when $0.5 \mathrm{~kg}$ of the TMR supplied. It is assumed that in case the particles were relatively small and the density was high, the feeder discharged them quickly. Consequently, when the feeding amount of TMR is small, the accuracy could be decreased because of the driving inertia of the discharging device.

The final prototype of TMR feeder developed in this study was suitable for feeding grow-finish pigs because the feeder performed stably with over $5.0 \mathrm{~kg}$ feedings. However, the feeder showed a lack of accuracy for feeding sows because the minimum amount of each feed was $0.5 \mathrm{~kg}$. For that reason, the improvement of outlet structure for accurate feeding is needed.

\section{Conflict of Interest}

No potential conflict of interest relevant to this article was reported. 


\section{Acknowledgement}

This research was supported by "Research Program for Agricultural Science \& Technology Development (Project PJ907029)", National Academy of Agricultural Science, Rural Development Administration, Republic of Korea.

\section{References}

Cho, S. B. 2009. Making and using technology of winter barley silage for pigs. 2009 Annual report of National Institute of Animal Science: 50-55.

Cho, S. B. 2012. An analysis on the effect of TMR feeding for grow-finish pigs. 2012 Annual report of National Institute of Animal Science: 13-15 (Unpublished).

Gatel, F., F. Grosjean and J. Castaing. 1988. Feeding Value of Ensiled High-Moisture Maize Grain with Cob for Growing-Finishing Pigs. Animal Feed Science and Technology, 20(2):145-153.

Hyundai Research Institute. 2012. International grain price increase and domestic price in Korea.
Kim, Y. H., E. H. Jeong, S. D. Lee, H. J. Jang, D. W. Kim, S. J. Sa, J. C. Park and I. C. Kim. 2012. The effect of feeding IRG pellet for sow productivity. Proceeding of international symposium for Development engineering. 2012(1):112 (In Korean).

Kim, H. J., J. T. Hong, B. K. Yu, S. C. Kim, K. H. Choi and H. H. Chang. 2012. Development of an electronic sow liquid system using RFID (1). Journal of Biosystems Engineering. 37(6): 373-384 (In Korean, with English abstract).

Ministry of Agriculture, Forestry and Fisheries(Japan). 2012. Plan for echo feed.

National Institute of Animal Science. 2009. TMR center Handbook in Korea.

PELLON co., ltd. 2007. Pig husbandry. Pellon brochure: 8-9.

Shimazawa, K. 2009. Studies of high-quality pork production using local agricultural by-product. $\mathrm{PhD}$ thesis. Kagoshima, Japan. Kagoshima University. United graduate school of agricultural science.

WATTAgNet. 2008. Robot takes the strain out of strawing. Available at: http://www. wattagnet.com/4526.html (Pig international, 2006). 